\title{
Nochmals zur Frage nach dem konstanten Vorkommen des Zentralkanals des Glaskörpers.
}

\author{
Von \\ Dr. E. Schaaff \\ in Strassburg i, E.
}

Auf die von $W$ olfrum ${ }^{1}$ ) gegen meine letzte Veröffentlichung ${ }^{2}$ ) erhobenen Einwände ausführlich einzugehen halte ich für unnütz, weil Wolf rum die Hauptsache zu erwähnen vergessen hat.

Die Hauptsache ist aber die:

Die Behauptung Wolfrums, der Glaskörperkanal sei kein konstantes Gebilde, beruht auf nichts anderem als auf der Tatsache, dass er mittels seiner Methode den Kanal nur in seltenen Fällen darstellen konnte. Da ich aber mit eben dieser Methode den Kanal gerade so häufig fand wie mit der ursprünglich von Stilling angegebenen, so kann ich mir diese durchans entgegengesetzten Resultate nur dadurch erklaren, dass in Wolfrums Präparationsmethode irgendeine Fehlerquelle gelegen hat.
1) v. Graefe's Arch. Bd. LXXIII, 1.
2) v. Graefe's Arch. Bd. LXXI, 1.

\section{Druckfehlerberichtigungen:}

In der Arbeit Koster Gzn. "Zum besseren Verständnis des Astigmatismus" Bd. LXXIV sind folgende Druckfehler zu berichtigen:

S. 175. In Fig. III, Nr. 3 soll in der Mitte der horizontalen Linie nur ein Strich stehen. Der Satz auf S. 175 in Klanmern: (Die Mittelpunkte der Figuren stehen auf horizontalen Linien) soll wegfallen.

S. 186. Fig.VII, Nr. 2. Die Maximum- und Minimummeridiane sollen $45^{\circ}$ gegen die Vertikale geneigt sein. Nr. 3 von Fig. VII soll soweit gedreht werden, dass $v v$ vertikal steht.

\section{Berichtigung:}

Die beiden ophthalmoskopischen Skizzen in Dr. Blessigs Mitteilung „Ein Fall von Retino-chorioiditis juxtapapillaris usw."(Bd. LXXIV, S, 285 u. 286) sind im aufrechten Bilde wiedergegeben, nicht, wie die Übersehriften besagen, im i. B.! 